\title{
RIESZ TRANSFORMS ON GRAPHS FOR $1 \leq p \leq 2$
}

\author{
EMMANUEL RUSS
}

\begin{abstract}
We prove, for $1<p \leq 2$, the $L^{p}$-boundedness of Riesz transforms on graphs satisfying the doubling property and a on-diagonal estimate of the Markov kernel. In [6], Coulhon and Duong proved the analogous result on Riemannian manifolds. We follow closely Coulhon and Duong's work. However, the discrete setting creates difficulties which do not appear in [6].
\end{abstract}

\section{Introduction}

This paper deals with Riesz transforms on graphs endowed with suitable Markov kernels. In this setting, one may define a discrete gradient $\nabla$ and a "Laplace operator" $\Delta$. The issue is to know whether $\|\nabla f\|_{p}$ and $\left\|(I-P)^{-\frac{1}{2}} f\right\|_{p}$ are comparable uniformly in $f$. It is clear when $p=2$. The question arises when $p \neq 2$ and is equivalent to the $L^{p}$-continuity of the operator $\nabla \Delta^{-\frac{1}{2}}$, which is called the Riesz transform.

Let $\Gamma$ be a infinite graph, endowed with a measure $m$ satisfying

$$
\forall x \in \Gamma, m(x)>0 .
$$

We assume that $\Gamma$ is connected and locally uniformly finite, which means that

$$
\sup _{x \in \Gamma} N(x)<\infty
$$

where, for $x \in \Gamma, N(x)$ is the number of neighbours of $x$. We also assume that $\Gamma$ is endowed with its natural distance $d$.

Denote by $B(x, r)$ the closed ball of center $x$ and of radius $r$, and by $V(x, r)$ its volume. We assume that $\Gamma$ has the doubling property, i. e. there exists $C>0$ such that

$$
V(x, 2 r) \leq C V(x, r), \quad \forall x \in \Gamma, \quad r>0 .
$$

That property implies that there exists $D>0$ such that

$$
V(x, \theta r) \leq C \theta^{D} V(x, r), \quad \forall x \in \Gamma, \quad r>0, \theta>1 .
$$

Received November 24, 1999. 
Let $p$ be a Markov kernel on $\Gamma$, i. e. a non-negative map defined on $\Gamma \times \Gamma$ such that

$$
\sum_{y \in \Gamma} p(x, y)=1, \quad \forall x \in \Gamma .
$$

Assume that $p$ is reversible with respect to $m$, which means that

$$
m(x) p(x, y)=m(y) p(y, x) \text { for all } x, y \in \Gamma .
$$

We also assume that there exists $r_{0}>0$ such that

$$
p(x, y)=0 \text { whenever } d(x, y) \geq r_{0}
$$

and that

$$
\inf _{d(x, y) \leq 1} p(x, y)>0 .
$$

The iterated kernel $p_{k}$ is defined by

$$
p_{k}(x, y)=\sum_{z} p_{k-1}(x, z) p(z, y) .
$$

Assume that the following upper estimate holds for $p_{k}$ : there exists $C>0$ such that

$$
p_{k}(x, x) \leq \frac{C m(x)}{V(x, \sqrt{k})}, \quad \forall k \in \mathrm{N}^{*}, \quad x \in \Gamma .
$$

This upper estimate, together with the doubling property, implies a Gaussian upper bound for $p_{k}$. Namely, there exists $\alpha>0$ and $C_{\alpha}>0$ such that, for any $k \in \mathrm{N}^{*}$ and any $x, y \in \Gamma$,

$$
p_{k}(x, y) \leq \frac{C_{\alpha} m(y)}{V(x, \sqrt{k})} \exp \left[-\alpha \frac{d^{2}(x, y)}{k}\right] .
$$

This is shown by Coulhon and Grigor'yan in [8], Theorem 1.1. More precisely, define, if $\Omega \subset \Gamma$ is finite and non-empty,

$$
\lambda_{1}(\Omega)=\inf \left\{\frac{\|\nabla f\|_{2}^{2}}{\|f\|_{2}^{2}} ; f \in c_{0}(\Gamma)\right\},
$$

where $c_{0}(\Gamma)$ is the set of all real-valued functions defined on $\Gamma$ and supported in $\Omega$. Then, say that $\Gamma$ satisfies a relative Faber-Krahn inequality if there exists $a>0, \nu>0$ such that, for any $x \in \Gamma$, any $r \geq \frac{1}{2}$ and any $\Omega \subset B(x, r)$, finite and non-empty, 


$$
\lambda_{1}(\Omega) \geq \frac{a}{r^{2}}\left[\frac{V(x, r)}{m(\Omega)}\right]^{\nu} .
$$

Then, Theorem 1.1 in [8] states the equivalence between:

1) the doubling property together with (7),

2) the doubling property together with (8),

3) the relative Faber-Krahn inequality.

The linear operator $P$ is defined by

$$
P f(x)=\sum_{y} p(x, y) f(y)
$$

and we notice that

$$
P^{k} f(x)=\sum_{y} p_{k}(x, y) f(y) .
$$

The reversibility assumption means that $P$ is self-adjoint on $L^{2}(\Gamma, m(x))$.

Denote by $\|f\|_{p}$ the $L^{p}$ norm of a function $f$, that is to say

$$
\|f\|_{p}=\left[\sum_{x}|f(x)|^{p} m(x)\right]^{\frac{1}{p}}
$$

and by $\|f\|_{1, \infty}$ the quantity

$$
\|f\|_{1, \infty}=\sup _{\lambda>0} \lambda m(\{x \in \Gamma \backslash|f(x)|>\lambda\}) .
$$

Notice that $L^{p}(\Gamma) \subset L^{q}(\Gamma)$ whenever $1 \leq p<q \leq \infty$.

The gradient of a function $f$ is defined by

$$
\nabla f(x)=\left[\frac{1}{2} \sum_{y} p(x, y)|f(y)-f(x)|^{2}\right]^{\frac{1}{2}}
$$

where the sum may be restricted to the ball $B\left(x, r_{0}\right)$.

The Riesz transform $T$ is defined as

$$
T=\nabla(I-P)^{-\frac{1}{2}}
$$

where the unbounded linear operator $(I-P)^{-\frac{1}{2}}$ is defined by means of spectral theory. Notice that $T$ is a subadditive operator, which means that

$$
|T(f+g)(x)| \leq|T f(x)|+|T g(x)| .
$$

Indeed, $(I-P)^{-\frac{1}{2}}$ is linear and $\nabla$ is subadditive.

We intend to show the following result: 
THEOREM 1. Let $\Gamma$ be a connected, infinite, locally uniformly finite graph. Assume that $\Gamma$ has the doubling property. Let $p$ be a reversible Markov kernel satisfying (5) and (6). Assume also the on-diagonal upper estimate (7). Then the Riesz transform $T$ is weak $(1,1)$ and bounded on $L^{p}$ if $1<p \leq 2$, which means that for all $p \in[1,2]$, there exists $C_{p}>0$ such that $\forall f$ with finite support,

$$
\|\nabla f\|_{p} \leq C_{p}\left\|(I-P)^{\frac{1}{2}} f\right\|_{p}, \quad 1<p \leq 2
$$

and, $\forall f$ with finite support,

$$
\|\nabla f\|_{1, \infty} \leq C_{1}\left\|(I-P)^{\frac{1}{2}} f\right\|_{1} .
$$

A result about discrete Riesz transforms is obtained by Hebisch and Saloff-Coste in [13]. They prove the $L^{p}$ boundedness of Riesz transforms on finitely generated groups with polynomial growth, for $1<p \leq 2$, by using Calderon-Zygmund theory on spaces of homogeneous type, but their approach fails to get such a result when $2<p<\infty$.

An analogous approach is not suitable for the proof of Theorem 1. Indeed, it would require a pointwise estimate for the gradient of $p_{k}(x, y)$. Such an estimate is false in general, because, in conjunction with the Gaussian upper estimate, it would imply a Gaussian lower bound for $p_{k}$ which does not hold under the assumptions of Theorem 1. The strategy of the proof is much inspired by [6], where Coulhon and Duong show that, for $1<p \leq 2$, the Riesz transforms are $L^{p}$-bounded on Riemannian manifolds with the doubling property and an on-diagonal upper bound of the heat kernel. First, we notice the $L^{2}$ continuity. Then, we use the Calderon-Zygmund decomposition in order to get that Riesz transforms are of weak type $(1,1)$. We conclude by interpolation.

In [6], Coulhon and Duong give a counterexample for the $L^{p}$ boundedness of the Riesz transform on Riemannian manifolds under the same assumptions when $p>2$. In section 4 , we shall prove that the analogous counterexample (i. e. two copies of $Z^{n}$ linked together by an edge) also works in our discrete setting.

When (9) holds, one may naturally wonder if $\left\|(I-P)^{\frac{1}{2}} f\right\|_{q}$ can be controlled by $\|\nabla f\|_{q}$ where $q$ is the conjugate exponent of $p$. In the continuous setting, it is well-known that, for every $p \in] 1,+\infty[$, the inequality

$$
\|\nabla f\|_{p} \leq C_{p}\left\|\Delta^{\frac{1}{2}} f\right\|_{p}, \quad \forall f \in C_{0}^{\infty}(M)
$$

implies by duality that

$$
\left\|\Delta^{\frac{1}{2}} f\right\|_{q} \leq C_{q}^{\prime}\|\nabla f\|_{q}, \quad \forall f \in C_{0}^{\infty}(M)
$$


where $\frac{1}{p}+\frac{1}{q}=1$. For a proof of this fact, see, for instance, [1] or [2]. In our discrete setting, we have a corresponding result. For every $p \in] 1,+\infty[$, the inequality

$$
\|\nabla f\|_{p} \leq C_{p}\left\|(I-P)^{\frac{1}{2}} f\right\|_{p}, \quad \forall f \in L^{p}(\Gamma) \cap L^{2}(\Gamma)
$$

implies that

$$
\left\|(I-P)^{\frac{1}{2}} f\right\|_{q} \leq C_{q}^{\prime}\|\nabla f\|_{q}, \quad \forall f \in L^{q}(\Gamma) \cap L^{2}(\Gamma)
$$

where $\frac{1}{p}+\frac{1}{q}=1$.

In order to prove that (10) implies (11), we will follow Bakry's ideas for the continuous setting. Let us start with the following lemma:

Lemma 1. Let $\left(T_{t}\right)_{t \geq 0}$ be a continuous semigroup of self-adjoint contracting operators on $L^{2}(X)$, where $(X, \mu)$ is a $\sigma$-finite measured space. Assume also that $\left(T_{t}\right)_{t>0}$ is a continuous semi-group on $L^{p}(X)$ for every $p \in[1,+\infty]$, and that $T_{t}$ contracts $L^{1}(X)$ and $L^{\infty}(X)$. Denote by $A$ the infinitesimal generator of $T_{t}$, and define $L_{0}^{p}$ as $L^{p} \cap(\operatorname{ker} A)^{\perp}$ (the orthogonal space of $\operatorname{ker}(A)$ in $L^{2}(X)$ ), for $1 \leq p \leq \infty$. Then, for $1<p<\infty,\left\{\left(A f \backslash f \in L^{p}(X) \cap D_{p}(A)\right\}\right.$ is dense in $L_{0}^{1}(X) \cap L^{\infty}(X)$ for the $L^{p}$ norm $\left(D_{p}(A)\right.$ is the domain of $A$ in $\left.L^{p}(X)\right)$.

The proof of this lemma will be given in an appendix.

Assume now that (10) holds and take $f \in L^{q}(\Gamma)$. We may write that

$$
\left\|(I-P)^{\frac{1}{2}} f\right\|_{q}=\sup _{g \in L^{p}, \mid g \|_{p} \leq 1}\left|\left\langle(I-P)^{\frac{1}{2}} f, g\right\rangle\right| .
$$

Define now

$$
\begin{aligned}
A & =-(I-P)^{\frac{1}{2}} \\
& =-\sum_{k=0}^{\infty} a_{k} P^{k}
\end{aligned}
$$

where the $a_{k}$ 's are given by

$$
(1-x)^{\frac{1}{2}}=\sum_{k=0}^{\infty} a_{k} x^{k} .
$$

The operator $A$ generates a semigroup which satisfies the requirements of Lemma 1. Indeed, if $S=I-(I-P)^{\frac{1}{2}}$, one has $S=-\sum_{k=1}^{\infty} a_{k} P^{k}$, so that 


$$
\begin{aligned}
\|S\|_{p \rightarrow p} & \leq \sum_{k=1}^{\infty}\left(-a_{k}\right)\left\|P^{k}\right\|_{p \rightarrow p} \\
& \leq 1
\end{aligned}
$$

It follows that

$$
\begin{aligned}
\left\|e^{-t(I-P)^{\frac{1}{2}}}\right\|_{p \rightarrow p} & \leq e^{-t} \sum_{k=0}^{\infty} \frac{t^{k}}{k !}\left\|S^{k}\right\|_{p \rightarrow p} \\
& \leq 1 .
\end{aligned}
$$

Moreover, $A$ is injective on $L^{2}(X)$, so that $\operatorname{ker}(A)=0$, and $D_{p}(A)=L^{p}(A)$. Therefore, thanks to Lemma $1, \quad\left\{(I-P)^{\frac{1}{2}} f \backslash f \in L^{p}(\Gamma)\right\}$ is dense in $L^{1}(\Gamma) \cap L^{\infty}(\Gamma)$, which is dense in $L^{p}(\Gamma)$. Hence, the supremum in (12) may be taken over $\left\{(I-P)^{\frac{1}{2}} h \backslash h \in L^{p}(\Gamma)\right\}$. If $h \in L^{p}(\Gamma)$ is such that $\left\|(I-P)^{\frac{1}{2}} h\right\|_{p} \leq 1$, one has

$$
\begin{aligned}
\left|\left\langle(I-P)^{\frac{1}{2}} f,(I-P)^{\frac{1}{2}} h\right\rangle\right| & =|\langle(I-P) f, h\rangle| \\
& =\left|\sum_{x, y} m(x) p(x, y)[f(x)-f(y)] h(x)\right| \\
& =\frac{1}{2}\left|\sum_{x, y} m(x) p(x, y)[f(x)-f(y)][h(x)-h(y)]\right| \\
& \leq \frac{1}{2} \sum_{x} m(x) \sum_{y} p(x, y)|f(y)-f(x)||h(y)-h(x)| \\
& \leq \frac{1}{2} \sum_{x} m(x)\left[\sum_{y} p(x, y)|f(y)-f(x)|^{2}\right]^{\frac{1}{2}} \\
& \times\left[\sum_{y} p(x, y)|h(y)-h(x)|^{2}\right]^{\frac{1}{2}} .
\end{aligned}
$$

Since $h \in L^{p}(\Gamma)$, we find that

$$
\begin{aligned}
\left|\left\langle(I-P)^{\frac{1}{2}} f,(I-P)^{\frac{1}{2}} h\right\rangle\right| & \leq C \sum_{x} m(x) \nabla f(x) \nabla h(x) \\
& \leq C\|\nabla f\|_{q}\|\nabla h\|_{p} .
\end{aligned}
$$

It follows that 


$$
\begin{aligned}
\left|\left\langle(I-P)^{\frac{1}{2}} f,(I-P)^{\frac{1}{2}} h\right\rangle\right| & =C\|\nabla f\|_{q}\|\nabla h\|_{p} \\
& \leq K C_{p}\|\nabla f\|_{q}\left\|(I-P)^{\frac{1}{2}} h\right\|_{p} \\
& \leq C_{p}^{\prime}\|\nabla f\|_{q} .
\end{aligned}
$$

The second line follows from our assumption (10), and the third is true because $\left\|(I-P)^{\frac{1}{2}} h\right\|_{p} \leq 1$. Finally, (11) holds.

We now turn to the proof of Theorem 1.

\section{Two results in view of Theorem 1}

\subsection{The Calderon-Zygmund decomposition}

We will need the following result, called the Calderon-Zygmund decomposition:

THeOREM 2. There exists $C>0$ such that, for any $f \in L^{1}(\Gamma) \cap L^{2}(\Gamma)$ and $\lambda>0$, one may write $f=g+b$ with $b=\sum_{i} b_{i}$ so that

a) $\forall x \in \Gamma,|g(x)| \leq C \lambda$.

b) $\forall i, \quad \exists B_{i}=B\left(x_{i}, r_{i}\right)$ so that the support of $b_{i}$ is contained in $B_{i}$, $\sum_{x}\left|b_{i}(x)\right| \leq C \lambda\left|B_{i}\right|$ and $\sum_{x} b_{i}(x)=0$.

c) $\sum_{i}\left|B_{i}\right| \leq \frac{C}{\lambda}\|f\|_{1}$.

d) $\exists k \in \mathbf{N}^{*}$ such that every $x \in \Gamma$ belongs at most to $k$ balls $B_{i}$.

For a proof in the general setting of homogeneous spaces, see [10]. Thanks to conditions $b$ ) and c), we see that

$$
\|b\|_{1} \leq \sum_{i}\left\|b_{i}\right\|_{1} \leq C \lambda \sum_{i}\left|B_{i}\right| \leq C\|f\|_{1}
$$

so that

$$
\|g\|_{1}=\|f-b\|_{1} \leq(1+C)\|f\|_{1} .
$$

\subsection{Estimates for the kernels}

We will also need the following lemma:

Lemma 2. There exists $\beta>0$ such that, for any $x \in \Gamma, l \in \mathrm{N}, k \in \mathrm{N}^{*}$, $\sum_{y \notin B(x, \sqrt{l})}\left|\nabla_{y} p_{k}(y, x)\right| m(y) \leq C m(x) e^{-\frac{\beta l}{k}} k^{-\frac{1}{2}}$.

Lemma 2 will follow from a few technical results.

Lemma 3. For all $\gamma>0, x \in \Gamma, l \in \mathrm{N}$ and $k \in \mathrm{N}^{*}$,

$\sum_{y \notin B(x, \sqrt{l})} e^{-2 \gamma \frac{d^{2}(x, y)}{k}} m(y) \leq C_{\gamma} V(x, \sqrt{k}) e^{-\frac{\gamma l}{k}}$. 
Indeed,

$$
\begin{aligned}
\sum_{y \notin B(x, \sqrt{l})} e^{-2 \gamma \frac{d^{2}(x, y)}{k}} m(y) & \leq e^{-\gamma \frac{l}{k}} \sum_{y} e^{-\gamma \frac{d^{2}(x, y)}{k}} m(y) \\
& \leq e^{-\gamma \frac{l}{k}} \sum_{i=0}^{\infty} \sum_{i \sqrt{k} \leq d(y, x)<(i+1) \sqrt{k}} e^{-\gamma^{\frac{d^{2}(y, x)}{k}}} m(y) \\
& \leq e^{-\gamma \frac{l}{k}} \sum_{i=0}^{\infty} e^{-\gamma i^{2}} V(x,(i+1) \sqrt{k}) \\
& \leq C e^{-\gamma \frac{l}{k}} \sum_{i=0}^{\infty} e^{-\gamma i^{2}}(i+1)^{D} V(x, \sqrt{k}) \\
& =C V(x, \sqrt{k}) e^{-\gamma \frac{l}{k}}
\end{aligned}
$$

The last but one line follows from (3).

As a consequence of Lemma 3 and (8) with $k=0$, we get

LeMmA 4. $\left.\sum_{y}\left[p_{k}(y, x)\right]^{2} e^{\frac{\gamma d^{2}(x, y)}{k}} m(y) \leq \frac{C_{\gamma} m^{2}(x)}{V(x, \sqrt{k})} \forall \gamma \in\right] 0,2 \alpha\left[, k \in \mathrm{N}^{*}, x \in \Gamma\right.$.

We now seek for a result analogous to Lemma 4 , replacing $p_{k}$ by its gradient. In order to get it, we will use the following result, which depends on (7) and (6):

LeMma 5. $\left|p_{k+1}(x, y)-p_{k}(x, y)\right| \leq \frac{C_{\alpha}^{\prime} m(y)}{k V^{\frac{1}{2}}(x, \sqrt{k}) V^{\frac{1}{2}}(y, \sqrt{k})}$.

The main tool used to get this estimate is the following statement:

Lemma 6. $\left\|(I-P)\left[\frac{p_{k}(x, .)}{m}\right]\right\|_{2} \leq \frac{C}{k V^{\frac{1}{2}}(x, \sqrt{k})}$.

We first consider the case where $k=2 l$. We write that

$$
(I-P)\left[\frac{p_{k}(x, .)}{m}\right]=(I-P) P^{l}\left[\frac{p_{l}(x, .)}{m}\right] .
$$

Indeed, 


$$
\begin{aligned}
P^{l}\left[\frac{p_{l}(x, .)}{m}\right](y) & =\sum_{z} p_{l}(y, z) \frac{p_{l}(x, z)}{m(z)} \\
& =\sum_{z} p_{l}(z, y) \frac{p_{l}(x, z)}{m(y)} \\
& =\frac{p_{k}(x, y)}{m(y)} .
\end{aligned}
$$

It follows from (14) that

$$
\left\|(I-P)\left[\frac{p_{k}(x, .)}{m}\right]\right\|_{2} \leq\left\|(I-P) P^{l}\right\|_{2 \rightarrow 2}\left\|\frac{p_{l}(x, .)}{m}\right\|_{2} .
$$

On the one hand,

$$
\begin{aligned}
\left\|\frac{p_{l}(x, .)}{m}\right\|_{2}^{2} & =\sum_{y} \frac{p_{l}(x, y) p_{l}(x, y)}{m(y)} \\
& =\frac{p_{k}(x, x)}{m(x)} \\
& \leq \frac{C}{V(x, \sqrt{k})} .
\end{aligned}
$$

On the other hand, if

$$
P=\int_{a}^{1} \lambda d E_{\lambda}
$$

where $a>-1$, we get that

$$
\begin{aligned}
\left\|(I-P) P^{l}\right\|_{2 \rightarrow 2} & \leq \sup _{\lambda \in[a, 1]}(1-\lambda)|\lambda|^{l} \\
& \leq \frac{C}{l}=\frac{2 C}{k} .
\end{aligned}
$$

We do the same when $k=l+(l+1)$. Therefore, Lemma 6 is proved.

REMARK. In this proof, we used the fact that $-1 \notin \mathrm{Sp}(P)$, which is implied by the on-diagonal lower bound of $p$, (6). Indeed, for any function $f \in L^{2}$, an elementary computation shows that 


$$
\begin{aligned}
\langle(I+P) f, f\rangle & =\frac{1}{2} \sum_{x, y}|f(x)+f(y)|^{2} p(x, y) m(x) \\
& \geq 2 \sum_{x}|f(x)|^{2} p(x, x) m(x) \\
& \geq c\|f\|_{2}^{2}
\end{aligned}
$$

where $c>0$. The role of $P$ 's spectrum with respect to the analyticity of $P$ on $L^{2}$ (see (15)) is pointed out in [9], Proposition 3, which claims that either $-1 \in \operatorname{Sp}(P)$ and one has $\left\|P^{k}-P^{k+1}\right\|_{2 \rightarrow 2}=2$ for all $k \in \mathrm{N}$, or $-1 \notin \operatorname{Sp}(P)$ and $P$ is analytic on $L^{2}$, which exactly means (15).

Let us deduce Lemma 5 from Lemma 6. Choose an integer $l \sim \frac{k}{2}$. One has

$$
\begin{aligned}
\left|p_{k+1}(x, y)-p_{k}(x, y)\right| & \leq \sum_{z} \frac{\left|p_{l+1}(x, z)-p_{l}(x, z)\right|}{m(z)} p_{l}(z, y) m(z) \\
& \leq\left\|\frac{p_{l+1}(x, .)-p_{l}(x, .)}{m}\right\|_{2}\left\|p_{l}(., y)\right\|_{2} .
\end{aligned}
$$

On the one hand, according to Lemma 6,

$$
\begin{aligned}
\left\|\frac{p_{l+1}(x, .)-p_{l}(x, .)}{m}\right\|_{2} & =\left\|(I-P)\left[\frac{p_{l}(x, .)}{m}\right]\right\|_{2} \\
& \leq \frac{C}{l V^{\frac{1}{2}}(x, \sqrt{l})} \\
& \leq \frac{C}{k V^{\frac{1}{2}}(x, \sqrt{k})},
\end{aligned}
$$

the last line being valid thanks to the doubling property. On the other hand,

$$
\begin{aligned}
\left\|p_{l}(., y)\right\|_{2}^{2} & =p_{2 l}(y, y) m(y) \\
& \leq \frac{C m^{2}(y)}{V(y, \sqrt{2 l})} \\
& \leq \frac{C m^{2}(y)}{V(y, \sqrt{k})}
\end{aligned}
$$

Thus, Lemma 5 is proved.

REMARK. The question naturally arises to know if this estimate about the 
temporal difference may be improved, and, namely, if one can get a Gaussian estimate:

$$
\left|p_{k+1}(x, y)-p_{k}(x, y)\right| \leq \frac{C m(y)}{k V(x, \sqrt{k})} \exp \left[-c \frac{d^{2}(x, y)}{k}\right] .
$$

In the continuous setting, the analogous estimate, i. e. an estimate on time derivatives of the heat kernel, follows rather easily from the on-diagonal estimate. In a discrete setting, the proof of (16) was given by Christ in [5] in the case of a polynomial volume growth. Christ's proof, which is difficult, may be adapted to the case of the doubling property, so that (16) does hold. But it is unnecessary for our purpose.

LEMmA 7. $\left.\sum_{y}\left|\nabla_{y} p_{k}(y, x)\right|^{2} e^{\frac{\gamma d^{2}(x, y)}{k}} m(y) \leq \frac{C_{\gamma} m^{2}(x)}{k V(x, \sqrt{k})}, \forall \gamma \in\right] 0, \alpha\left[, k \in \mathrm{N}^{*}\right.$.

Define

$$
I(k, x)=\sum_{y}\left|\nabla_{y} p_{k}(y, x)\right|^{2} e^{\frac{d d^{2}(x, y)}{k}} m(y) .
$$

The very definition of $\nabla$ shows that

$$
I(k, x)=\frac{1}{2} \sum_{y, z}\left|p_{k}(y, x)-p_{k}(z, x)\right|^{2} e^{\gamma^{d^{2}(x, y)}} \frac{1}{k} m(y) .
$$

Remember that this sum may be restricted to the $(y, z)$ such that $d(y, z) \leq r_{0}$, since $p(y, z)=0$ if it is not the case. To estimate the analogous quantity in a continuous setting, Grigor'yan, in [12], Theorem 1.1, makes several integrations by parts. Such computations do not work very well in a discrete setting. We replace them by computations about $I(k, x)$ inspired by the proof of the estimate of $\nabla p^{(n)}$ in Theorem 5.1 of [13], and using the temporal estimate given by Lemma 5 :

$$
\begin{aligned}
I(k, x) & =\sum_{d(y, z) \leq r_{0}} p_{k}(y, x)\left[p_{k}(y, x)-p_{k}(z, x)\right] m(y) p(y, z) e^{\gamma^{\frac{d^{2}(x, y)}{k}}} \\
& -\sum_{d(y, z) \leq r_{0}} p_{k}(z, x)\left[p_{k}(y, x)-p_{k}(z, x)\right] m(y) p(y, z) e^{\frac{d^{2}(x, y)}{k}} \\
& =\sum_{d(y, z) \leq r_{0}} p_{k}(y, x)\left[p_{k}(y, x)-p_{k}(z, x)\right] m(y) p(y, z) e^{\gamma^{\frac{d^{2}(x, y)}{k}}} \\
& +\sum_{d(y, z) \leq r_{0}} p_{k}(y, x)\left[p_{k}(y, x)-p_{k}(z, x)\right] m(y) p(y, z) e^{\frac{d^{\frac{d^{2}(x, z)}{k}}}{k}}
\end{aligned}
$$


In the last line, we inverted $y$ and $z$ and we used the reversibility of $p$. Hence, we get that

$$
\begin{aligned}
I(k, x) & \leq 2 \sum_{d(y, z) \leq r_{0}} p_{k}(y, x)\left[p_{k}(y, x)-p_{k}(z, x)\right] m(y) p(y, z) e^{\gamma \frac{d^{2}(x, y)}{k}} \\
& +\sum_{d(y, z) \leq r_{0}} p_{k}(y, x)\left[p_{k}(y, x)-p_{k}(z, x)\right] m(y) p(y, z)\left[e^{\gamma \frac{d^{2}(x, z)}{k}}-e^{\gamma \frac{d^{2}(x, y)}{k}}\right] \\
& =2 I_{1}(k, x)+I_{2}(k, x) .
\end{aligned}
$$

Thanks to the preceeding lemma, we can estimate $I_{1}$ and $I_{2}$. Indeed,

$$
\begin{aligned}
I_{1}(k, x) & =\sum_{y} p_{k}(y, x) e^{\gamma \frac{d^{2}(x, y)}{k}} m(y) \sum_{z} p(y, z)\left[p_{k}(y, x)-p_{k}(z, x)\right] \\
& =\sum_{y} p_{k}(y, x) e^{\gamma \frac{d^{2}(x, y)}{k}} m(y)\left[p_{k}(y, x)-p_{k+1}(y, x)\right]
\end{aligned}
$$

hence

$$
\begin{aligned}
\left|I_{1}(k, x)\right| & \leq \frac{C_{\alpha}^{\prime} m(x)}{k V^{\frac{1}{2}}(x, \sqrt{k})} \sum_{y} \frac{p_{k}(y, x)}{V^{\frac{1}{2}}(y, \sqrt{k})} e^{\gamma^{d^{2}(x, y)}} m(y) \\
& \leq \frac{C_{\alpha}^{\prime} C_{\alpha} m^{2}(x)}{k V^{\frac{3}{2}}(x, \sqrt{k})} \sum_{y} \frac{1}{V^{\frac{1}{2}}(y, \sqrt{k})} e^{(\gamma-\alpha) \frac{d^{2}(x, y)}{k}} m(y) \\
& \leq \frac{C_{\alpha} C_{\alpha}^{\prime} C_{\gamma} m^{2}(x)}{k V^{2}(x, \sqrt{k})} \sum_{y} e^{\left(\gamma^{\prime}-\alpha\right) \frac{d^{2}(x, y)}{k}} m(y) \\
& \leq \frac{C_{\gamma} m^{2}(x)}{k V(x, \sqrt{k})} .
\end{aligned}
$$

The first line holds thanks to Lemma 5, the last one follows from Lemma 3. Note that, in this computation, it is possible to choose $\left.\gamma^{\prime} \in\right] \gamma, \alpha[$ because $\gamma<\alpha$.

As for $I_{2}$, denote by $f$ the map defined by

$$
f(x)=e^{\frac{\gamma}{k} x^{2}} .
$$

We may write, according to the mean-value theorem, that

$$
|f(b)-f(a)| \leq \frac{2 \gamma}{k}|b-a| \sup (a, b) e^{\frac{\gamma}{k} \sup (a, b)^{2}} .
$$

Applying this inequality with $a=d(x, y), b=d(x, z)$ when $d(y, z) \leq r_{0}$, so that $|d(x, y)-d(x, z)| \leq r_{0}$, we get, if we notice that $\sup (a, b) \leq a+r_{0}$, that 


$$
\begin{aligned}
& \left|I_{2}(k, x)\right| \leq \frac{2 \gamma}{k} \sum_{d(y, z) \leq r_{0}} m(y) p(y, z) p_{k}(y, x) \mid p_{k}(y, x) \\
& -p_{k}(z, x) \mid\left[d(x, y)+r_{0}\right]^{\left.e^{\frac{\gamma}{k}} d(x, y)+r_{0}\right]^{2}} \\
& =2 \sqrt{\frac{\gamma}{k}} \sum_{d(y, z) \leq r_{0}} m(y) p(y, z) p_{k}(y, x) \mid p_{k}(y, x) \\
& -p_{k}(z, x) \mid \sqrt{\frac{\gamma}{k}\left[d(x, y)+r_{0}\right]^{2}} e^{\left.\frac{\gamma}{k} d(x, y)+r_{0}\right]^{2}} \\
& \leq 2 \sqrt{\frac{\gamma}{k}} \sum_{d(y, z) \leq r_{0}} m(y) p(y, z) p_{k}(y, x)\left|p_{k}(y, x)-p_{k}(z, x)\right| e^{\frac{\gamma^{\prime}}{k}\left[(d x, y)+r_{0}\right]^{2}} \\
& \leq 2 \sqrt{\frac{\gamma}{k}}\left[\sum_{d(y, z) \leq r_{0}} m(y) p(y, z)\left[p_{k}(y, x)\right]^{2} e^{\frac{\gamma^{\prime \prime}}{k}\left[d(x, y)+r_{0}\right]^{2}}\right]^{\frac{1}{2}} \\
& \times\left[\sum_{d(y, z) \leq r_{0}} m(y) p(y, z)\left|p_{k}(y, x)-p_{k}(z, x)\right|^{2} e^{\left.\frac{\gamma}{k} d(x, y)+r_{0}\right]^{2}}\right]^{\frac{1}{2}} .
\end{aligned}
$$

But

$$
\frac{\gamma}{k}\left[d(x, y)+r_{0}\right]^{2}=\frac{\gamma}{k}\left[d^{2}(x, y)+2 r_{0} d(x, y)+r_{0}^{2}\right]
$$

and, in the sums which define $I(k, x)$, we may assume that $d(x, y) \leq(k+1) r_{0}$ and that $d(x, z) \leq(k+1) r_{0}$. If it was not the case, since $d(y, z) \leq r_{0}$, we would obtain $d(x, y)>k r_{0}$ and $d(x, z)>k r_{0}$, so $p_{k}(y, x)=p_{k}(z, x)=0$ according to (5). Finally,

$$
\frac{\gamma}{k}\left[d(x, y)+r_{0}\right]^{2} \leq \frac{\gamma}{k} d^{2}(x, y)+C
$$

and we can write that

$$
\begin{aligned}
\left|I_{2}(k, x)\right| & \leq C \sqrt{\frac{\gamma}{k}}\left[\sum_{y}\left[p_{k}(y, x)\right]^{2} e^{\gamma^{\gamma^{\prime d^{2}(x, y)}}} m(y)\right]^{\frac{1}{2}} \sqrt{I(k, x)} \\
& \leq \frac{C_{\gamma}}{\sqrt{k}} \frac{m(x)}{\sqrt{V(x, \sqrt{k})}} \sqrt{I(k, x)} .
\end{aligned}
$$

If we use simultaneously the estimates about $I_{1}$ and $I_{2}$, we find that 


$$
I \leq \frac{C_{\gamma} m^{2}(x)}{k V(x, \sqrt{k})}+\frac{C_{\gamma} m(x)}{\sqrt{k V(x, \sqrt{k})}} \sqrt{I}
$$

from which we get the right estimate for $I$, hence Lemma 7 by (17).

Thanks to Lemma 7 and Lemma 3, we can finally show Lemma 2. Indeed, we just have to write that, if $\beta<\frac{\alpha}{4}$,

$$
\begin{aligned}
\sum_{y, d(y, x) \geq \sqrt{l}}\left|\nabla_{y} p_{k}(x, y)\right| m(y) & \leq\left[\sum_{y, d(y, x) \geq \sqrt{l}}\left|\nabla_{y} p_{k}(x, y)\right|^{2} e^{4 \beta^{\frac{d^{2}(x, y)}{k}}} m(y)\right]^{\frac{1}{2}} \\
& \times\left[\sum_{d(y, x) \geq \sqrt{l}} e^{\left.-4 \beta^{\frac{d^{2}(x, y)}{k}} m(y)\right]^{\frac{1}{2}}}\right. \\
& \leq \frac{C m(x)}{\sqrt{k V(x, \sqrt{k})}} \sqrt{V(x, \sqrt{k})} e^{-\frac{\beta l}{k}}
\end{aligned}
$$

which is Lemma 2.

\section{Proof of Theorem 1}

The proof follows closely [6]. The $L^{2}$ boundedness of $T$ is obvious. Indeed, if $f \in L^{2}(\Gamma)$,

$$
\nabla f(x)=\left[\frac{1}{2} \sum_{y} p(x, y)|f(y)-f(x)|^{2}\right]^{\frac{1}{2}}
$$

so that

$$
\begin{aligned}
\|\nabla f\|_{2}^{2} & \leq C \sum_{y, x} p(x, y)|f(x)-f(y)|^{2} m(x) \\
& =2 C\langle(I-P) f, f\rangle \\
& =2 C\left\|(I-P)^{\frac{1}{2}} f\right\|_{2}^{2} .
\end{aligned}
$$

If we show that $T$ is weak $(1,1)$, the Marcinkiewicz interpolation theorem will give the $L^{p}$ boundedness for $1<p<2$. Therefore, we are going to show that $T$ is weak $(1,1)$.

Let $f \in L^{1}(\Gamma) \cap L^{2}(\Gamma)$. Our aim is to show that, if $\lambda>0$,

$$
m(\{x \backslash|T f(x)|>\lambda\}) \leq \frac{C}{\lambda}\|f\|_{1} .
$$


Write the Calderon-Zygmund decomposition of $f$ :

$$
f=g+b=g+\sum_{i} b_{i}
$$

One has

$$
m(\{x \backslash|T f(x)|>\lambda\}) \leq m\left(\left\{x \backslash|\operatorname{Tg}(x)|>\frac{\lambda}{2}\right\}\right)+m\left(\left\{x \backslash|\operatorname{Tb}(x)|>\frac{\lambda}{2}\right\}\right)
$$

because $T$ is subadditive. Since $T$ is $L^{2}$ bounded, we may write that

$$
\begin{aligned}
m\left(\left\{x \backslash|T g(x)|>\frac{\lambda}{2}\right\}\right) & \leq \frac{4}{\lambda^{2}}\|T g\|_{2}^{2} \\
& \leq \frac{C}{\lambda^{2}}\|g\|_{2}^{2} \\
& \leq \frac{C}{\lambda^{2}} \lambda\|g\|_{1} \\
& \leq \frac{C}{\lambda}\|f\|_{1} .
\end{aligned}
$$

The last but one line is true because of the property $a$ ) of the decomposition.

Therefore, what remains to be proved is the fact that

$$
m(\{x \backslash|T b(x)|>\lambda\}) \leq \frac{C}{\lambda}\|f\|_{1} .
$$

To this purpose, we write that

$$
b=\sum_{i} b_{i}=\sum_{i} P^{k_{i}} b_{i}+\sum_{i}\left(I-P^{k_{i}}\right) b_{i}
$$

where $k_{i}=r_{i}^{2}$ if $b_{i}$ is supported in $B_{i}=B\left(x_{i}, r_{i}\right)$. First, we prove that

$$
\left\|\sum_{i} P^{k_{i}} b_{i}\right\|_{2}^{2} \leq C \lambda\|f\|_{1}
$$

One has

$$
\begin{aligned}
\left|P^{k_{i}} b_{i}(x)\right| & \leq \sum_{y} p_{k_{i}}(x, y)\left|b_{i}(y)\right| \\
& \leq C \frac{1}{V\left(x, r_{i}\right)} \sum_{y} \exp \left(-\alpha \frac{d^{2}(x, y)}{k_{i}}\right)\left|b_{i}(y)\right| m(y) .
\end{aligned}
$$

In the last sum, we can assume that $y \in B\left(x_{i}, r_{i}\right)$ because of the support of $b_{i}$. But, if $d\left(y, x_{i}\right)<r_{i}$, then $d\left(x, x_{i}\right) \leq d(x, y)+r_{i}$, hence $d^{2}\left(x, x_{i}\right) \leq 2 d^{2}(x, y)+$ 
$2 r_{i}^{2}$. It follows that

$$
\begin{aligned}
\left|P^{k_{i}} b_{i}(x)\right| & \leq \frac{C}{V\left(x, r_{i}\right)} \exp \left(-\alpha \frac{d^{2}\left(x, x_{i}\right)}{2 k_{i}}\right)\left\|b_{i}\right\|_{1} \\
& \leq \frac{C}{V\left(x, r_{i}\right)} \exp \left(-\alpha \frac{d^{2}\left(x, x_{i}\right)}{2 k_{i}}\right) \lambda m\left(B_{i}\right) \\
& \leq C \lambda \sum_{y} \frac{1}{V\left(x, r_{i}\right)} \exp \left(-\frac{\alpha}{2 k_{i}} d^{2}\left(x, x_{i}\right)\right) 1_{B_{i}}(y) m(y) \\
& \leq C \lambda \sum_{y} \frac{1}{V\left(x, r_{i}\right)} \exp \left(-\alpha \frac{d^{2}(x, y)}{4 k_{i}}\right) 1_{B_{i}}(y) m(y) .
\end{aligned}
$$

The second line follows from property $b$ ) of the decomposition. This inequality implies that

$$
\begin{aligned}
\left\|\sum_{i} P^{k_{i}} b_{i}\right\|_{2} & \leq\left\|\sum_{i} C \lambda \sum_{y} \frac{1}{V\left(., r_{i}\right)} \exp \left(-\alpha \frac{d^{2}(., y)}{k_{i}}\right) 1_{B_{i}}(y) m(y)\right\|_{2} \\
& \leq C \lambda\left\|\sum_{i} \sum_{y} \frac{1}{V\left(., r_{i}\right)} \exp \left(-\alpha \frac{d^{2}(., y)}{k_{i}}\right) 1_{B_{i}}(y) m(y)\right\|_{2} \\
& =C \lambda \sup _{\|f\|_{2}=1} \mid \sum_{x} \sum_{i} \sum_{y} \frac{1}{V\left(x, r_{i}\right)} \\
& \times \exp \left(-\alpha \frac{d^{2}(x, y)}{k_{i}}\right) 1_{B_{i}}(y) m(y) f(x) m(x) \mid .
\end{aligned}
$$

But, because of the doubling property,

$$
V\left(y, r_{i}\right) \leq\left[1+\frac{d(x, y)}{r_{i}}\right]^{D} V\left(x, r_{i}\right)
$$

(actually, $B\left(y, r_{i}\right) \subset B\left(x, r_{i}+d(x, y)\right)=B\left(x,\left[1+\frac{d(x, y)}{r_{i}}\right] r_{i}\right)$ and one may apply (3)). Thus, 


$$
\begin{aligned}
& \sum_{x} \frac{1}{V\left(x, r_{i}\right)} \exp \left(-\alpha \frac{d^{2}(x, y)}{k_{i}}\right)|f(x)| m(x) \leq \frac{1}{V\left(y, r_{i}\right)} \sum_{x}\left[1+\frac{d(x, y)}{r_{i}}\right]^{D} \\
& \times \exp \left(-\alpha \frac{d^{2}(x, y)}{k_{i}}\right)|f(x)| m(x) \leq \frac{1}{V\left(y, r_{i}\right)} \sum_{x} \exp \left(-\alpha^{\prime} \frac{d^{2}(x, y)}{k_{i}}\right)|f(x)| m(x) \\
& \leq \frac{1}{V\left(y, r_{i}\right)}\left[\sum_{l=0}^{\infty} \sum_{2^{2 l} k_{i} \leq d^{2}(x, y) \leq<2^{2 l+2} k_{i}} \exp \left(-\alpha \frac{d^{2}(x, y)}{k_{i}}\right)|f(x)| m(x)\right] \\
& +\frac{1}{V\left(y, r_{i}\right)} \sum_{d^{2}(x, y)<k_{i}} \exp \left(-\alpha \frac{d^{2}(x, y)}{k_{i}}\right)|f(x)| m(x) \leq \frac{1}{V\left(y, r_{i}\right)} \sum_{l=0}^{\infty} e^{-\alpha 2^{2 l}} \\
& \times \sum_{x \in B\left(y, 2^{l+1} r_{i}\right)}|f(x)| m(x)+\frac{1}{V\left(y, r_{i}\right)} \sum_{d(x, y)<r_{i}}|f(x)| m(x) .
\end{aligned}
$$

In the second line, we used the fact that $k_{i}=r_{i}^{2}$.

Hence, if we denote by

$$
M f(x)=\sup _{x \in B} \frac{1}{|B|} \sum_{z \in B}|f(z)| m(z)
$$

the Hardy-Littlewood maximal function of $f$, we get

$$
\begin{aligned}
\sum_{x} \frac{1}{V\left(x, r_{i}\right)} \exp \left(-\alpha \frac{d^{2}(x, y)}{r_{i}}\right)|f(x)| m(x) & \leq \sum_{l=0}^{\infty} e^{-\alpha 2^{2 l}} \frac{V\left(y, 2^{l+1} r_{i}\right)}{V\left(y, r_{i}\right)} \frac{1}{V\left(y, 2^{l+1} r_{i}\right)} \\
& \times \sum_{x \in B\left(y, 2^{l+1} r_{i}\right)}|f(x)| m(x) \\
& +\frac{1}{V\left(y, r_{i}\right)} \sum_{x \in B\left(y, r_{i}\right)}|f(x)| m(x) \\
& \leq M f(y)+\sum_{l=0}^{\infty} e^{-\alpha 2^{2 l}} 2^{D(l+1)} M f(y) \\
& =K M f(y) .
\end{aligned}
$$

Returning to (19), we find that 


$$
\begin{aligned}
\left\|\sum_{i} P^{k_{i}} b_{i}\right\|_{2} & \leq C \lambda \sup _{\|f\|_{2}=1} \sum_{y} M f(y) \sum_{i} 1_{B_{i}}(y) m(y) \\
& \leq C \lambda \sup _{\|f\|_{2}=1}\|M f\|_{2}\left\|\sum_{i} 1_{B_{i}}\right\|_{2} .
\end{aligned}
$$

But it is well-known that $\|M f\|_{2} \leq\|f\|_{2}$ (see [10] for a proof in the setting of homogeneous spaces), so that we obtain

$$
\begin{aligned}
\left\|\sum_{i} P^{k_{i}} b_{i}\right\|_{2}^{2} & \leq C \lambda^{2}\left\|\sum_{i} 1_{B_{i}}\right\|_{2}^{2} \\
& \leq C \lambda^{2}\left\|\sum_{i} 1_{B_{i}}\right\|_{\infty}\left\|\sum_{i} 1_{B_{i}}\right\|_{1} .
\end{aligned}
$$
We notice that, because of property $d), \sum_{i} 1_{B_{i}}(z)=\left|\left\{i \backslash z \in B_{i}\right\}\right| \leq k$. More-
over,

$$
\begin{aligned}
\left\|\sum_{i} 1_{B_{i}}\right\|_{1} & \leq \sum_{i}\left\|1_{B_{i}}\right\|_{1} \\
& \leq \sum_{i} V\left(B_{i}\right) \\
& \leq \frac{C}{\lambda}\|f\|_{1} .
\end{aligned}
$$

Property c) of the decomposition implies the last line.

We get

$$
\left\|\sum_{i} P^{k_{i}} b_{i}\right\|_{2}^{2} \leq C \lambda\|f\|_{1} .
$$

Finally, one has

$$
\begin{aligned}
\left.m\left(\{x\rangle\left|T\left[\sum_{i} P^{k_{i}} b_{i}\right](x)\right|>\frac{\lambda}{2}\right\}\right) & \leq \frac{4}{\lambda^{2}}\left\|T\left[\sum_{i} P^{k_{i}} b_{i}\right]\right\|_{2}^{2} \\
& \leq \frac{C}{\lambda^{2}}\left\|\sum_{i} P^{k_{i}} b_{i}\right\|_{2}^{2} \\
& \leq \frac{C}{\lambda}\|f\|_{1},
\end{aligned}
$$


where the $L^{2}$ boundedness of $T$ is used in the second line. has

We are now going to estimate $\left.m\left(\{x\rangle\left|T\left[\sum_{i}\left(I-P^{k_{i}}\right) b_{i}\right](x)\right|>\frac{\lambda}{2}\right\}\right)$. One (20) $\left.m\left(\{x\rangle\left|T\left[\sum_{i}\left(I-P^{k_{i}}\right) b_{i}\right]\right|>\frac{\lambda}{2}\right\}\right) \leq m\left(\left\{x \in \bigcup_{i} 2 B_{i}\right\rangle \mid\right.$

$$
\begin{aligned}
& \left.\left.T\left[\sum_{i}\left(I-P^{k_{i}}\right) b_{i}\right](x) \mid>\frac{\lambda}{2}\right\}\right) \\
+ & m\left(\left\{x \notin \bigcup_{i} 2 B_{i} \backslash \mid\right.\right. \\
& \left.\left.T\left[\sum_{i}\left(I-P^{k_{i}}\right) b_{i}\right](x) \mid>\frac{\lambda}{2}\right\}\right) \\
\leq & m\left(\bigcup_{i} 2 B_{i}\right)+m\left(\left\{x \notin \bigcup_{i} 2 B_{i} \backslash \mid\right.\right. \\
& \left.\left.T\left[\sum_{i}\left(I-P^{k_{i}}\right) b_{i}\right](x) \mid>\frac{\lambda}{2}\right\}\right) .
\end{aligned}
$$

As for the first term of (20),

$$
\begin{aligned}
m\left(\bigcup_{i} 2 B_{i}\right) & \leq \sum_{i} m\left(2 B_{i}\right) \\
& \leq C \sum_{i} m\left(B_{i}\right) \\
& \leq \frac{C}{\lambda}\|f\|_{1} .
\end{aligned}
$$

So, we shall deal with the second term of (20). 


$$
\begin{aligned}
& m\left(\left\{x \notin \bigcup_{i} 2 B_{i} \backslash\left|T\left[\sum_{i}\left(I-P^{k_{i}}\right) b_{i}\right](x)\right|>\frac{\lambda}{2}\right\}\right) \\
& \left.\leq \frac{2}{\lambda} \sum_{y \notin \bigcup_{i} 2 B_{i}}|T| \sum_{i}\left(I-P^{k_{i}}\right) b_{i}\right](y) \mid m(y) \\
& \leq \frac{2}{\lambda} \sum_{y \notin \bigcup_{i} 2 B_{i}} \sum_{i}\left|T\left(I-P^{k_{i}}\right) b_{i}(y)\right| m(y) .
\end{aligned}
$$

Let us prove that, for every $i$,

$$
\sum_{y \notin \bigcup_{j} 2 B_{j}}\left|T\left(I-P^{k_{i}}\right) b_{i}(y)\right| m(y) \leq C\left\|b_{i}\right\|_{1} .
$$

If we denote by $q_{k_{i}}$ the kernel of $T\left(I-P^{k_{i}}\right)$, we have

$$
\begin{aligned}
\sum_{y \notin \bigcup_{j} 2 B_{j}}\left|T\left(I-P^{k_{i}}\right) b_{i}(y)\right| m(y) & \leq \sum_{y \notin \bigcup_{j} 2 B_{j}} \sum_{x}\left|q_{k_{i}}(y, x)\right|\left|b_{i}(x)\right| m(y) \\
& =\sum_{x \in B_{i}}\left|b_{i}(x)\right| \sum_{y \notin \bigcup_{j} 2 B_{j}}\left|q_{k_{i}}(y, x)\right| m(y) .
\end{aligned}
$$

But, when $y \notin \bigcup_{j} 2 B_{j}, y \notin 2 B_{i}$, so that $d(y, x) \geq r_{i}=\sqrt{k_{i}}$. This implies that

$$
\sum_{y \notin \bigcup_{j} 2 B_{j}}\left|T\left(I-P^{k_{i}}\right) b_{i}(y)\right| m(y) \leq \sum_{x \in B_{i}}\left|b_{i}(x)\right| \sum_{d(y, x) \geq \sqrt{k_{i}}}\left|q_{k_{i}}(y, x)\right| m(y)
$$

and we just have to show that

$$
\sum_{d(y, x) \geq \sqrt{k_{i}}}\left|q_{k_{i}}(y, x)\right| m(y) \leq C m(x)
$$

where $C>0$ is independent of $x$, for, if this is proved, we will get

$$
\sum_{y \notin \bigcup_{j} 2 B_{j}}\left|T\left(I-P^{k_{i}}\right) b_{i}(y)\right| m(y) \leq C\left\|b_{i}\right\|_{1}
$$

as claimed.

Let us compute $q_{k}$, the kernel of $\nabla(I-P)^{-\frac{1}{2}}\left(I-P^{k}\right)$. If $\left(a_{p}\right)_{p \geq 0}$ is the sequence of real numbers defined by 
RIESZ TRANSFORMS ON GRAPHS FOR $1 \leq p \leq 2$

$$
(1-x)^{-\frac{1}{2}}=\sum_{p=0}^{\infty} a_{p} x^{p}
$$

we see that

$$
\begin{aligned}
(I-P)^{-\frac{1}{2}}\left(I-P^{k}\right) & =\sum_{p=0}^{\infty} a_{p} P^{p}\left(I-P^{k}\right) \\
& =\sum_{p=0}^{\infty} a_{p} P^{p}-\sum_{p=0}^{\infty} a_{p} P^{p+k} \\
& =\sum_{p=0}^{\infty}\left(a_{p}-b_{p}\right) P^{p}
\end{aligned}
$$

where

$$
b_{p}=\left\{\begin{array}{lll}
a_{p-k} & \text { if } & p \geq k \\
0 & \text { if } \quad & p<k
\end{array}\right.
$$

Hence,

$$
T\left(I-P^{k}\right)=\nabla\left[\sum_{p=0}^{\infty}\left(a_{p}-b_{p}\right) P^{p}\right]
$$

so that

$$
\left|q_{k}(x, y)\right| \leq \sum_{p=0}^{\infty}\left|a_{p}-b_{p}\right| \nabla_{x} p_{p}(x, y) .
$$

We find that

$$
\begin{aligned}
\sum_{d(y, x) \geq \sqrt{k}}\left|q_{k}(x, y)\right| m(x) & \leq \sum_{p=0}^{\infty}\left|a_{p}-b_{p}\right| \sum_{d(y, x) \geq \sqrt{k}} \nabla_{x} p^{p}(x, y) m(x) \\
& \leq\left[C+C \sum_{p=1}^{\infty}\left|a_{p}-b_{p}\right| e^{-\frac{\beta k}{p}} p^{-\frac{1}{2}}\right] m(y) \\
& \leq\left[C+C \sum_{p=1}^{k-1}\left|a_{p}\right| e^{-\frac{\beta k}{p}} p^{-\frac{1}{2}}+\sum_{p=k}^{\infty}\left|a_{p}-b_{p}\right| p^{-\frac{1}{2}}\right] m(y) .
\end{aligned}
$$

The upper bound given by Lemma 2 shows the second line.

It is well-known (by the Stirling formula) that 


$$
a_{p}=\frac{(2 p) !}{4^{p}(p !)^{2}} \sim \frac{1}{\sqrt{\pi p}} .
$$

Hence, we get that

$$
\sum_{p=1}^{k-1} a_{p} e^{-\frac{\beta k}{p}} p^{-\frac{1}{2}} \leq C \sum_{p=1}^{k-1} \frac{e^{-\frac{\beta k}{p}}}{p} .
$$

If we denote by $f$ the function

$$
f(x)=\frac{e^{-\frac{\beta k}{x}}}{x}
$$

we observe, considering $\log f$, that $f$ is nondecreasing on $[1, \beta k]$ and nonincreasing on $[\beta k, k-1]$, so that

$$
\sum_{p=1}^{E(\beta k)} \frac{e^{-\frac{\beta k}{p}}}{p} \leq \int_{1}^{E(\beta k)+1} \frac{e^{-\frac{\beta k}{x}}}{x} d x
$$

and

$$
\sum_{p=E(\beta k)+1}^{k-1} \frac{e^{-\frac{\beta k}{p}}}{p} \leq \int_{E(\beta k)}^{k-1} \frac{e^{-\frac{\beta k}{x}}}{x} d x .
$$

We get

$$
\sum_{p=1}^{k-1} \frac{e^{-\frac{\beta k}{p}}}{p} \leq 2 \int_{0}^{k} \frac{e^{-\frac{\beta k}{x}}}{x} d x=2 \int_{0}^{1} \frac{e^{-\frac{\beta}{u}}}{u} d u=\text { Cste. }
$$

As for the second term of (24), we write that

$$
a_{p}-a_{p+1}=2 \frac{(2 p) !(p+1)}{4^{p+1}[(p+1) !]^{2}} .
$$

The Stirling formula shows that

$$
a_{p}-a_{p+1} \sim \frac{C}{p^{\frac{3}{2}}} .
$$

So, there exists a constant $K$ such that

$$
\forall p \in \mathrm{N}^{*}, \quad a_{p}-a_{p+1} \leq \frac{K}{p^{\frac{3}{2}}} .
$$

For $p \geq k+2,\left|a_{p}-b_{p}\right|=\left|a_{p}-a_{p-k}\right|=a_{p-k}-a_{p}$, hence 


$$
\begin{aligned}
\left|a_{p}-b_{p}\right| & =\sum_{l=p-k}^{p-1}\left(a_{l}-a_{l+1}\right) \\
& \leq K \sum_{l=p-k}^{p-1} \frac{1}{l^{\frac{3}{2}}} \\
& \leq K \int_{p-k-1}^{p-1} \frac{d x}{x^{\frac{3}{2}}} \\
& =K\left[\frac{1}{\sqrt{p-k-1}}-\frac{1}{\sqrt{p-1}}\right] .
\end{aligned}
$$

This implies that

$$
\begin{aligned}
\sum_{p=k+2}^{\infty}\left|a_{p}-b_{p}\right| p^{-\frac{1}{2}} & \leq K \sum_{p=k+2}^{\infty}\left[\frac{1}{\sqrt{p-k-1}}-\frac{1}{\sqrt{p-1}}\right] \frac{1}{\sqrt{p}} \\
& \leq K \sum_{p=k+2}^{\infty}\left[\frac{k}{\sqrt{p-k-1} \sqrt{p-1}[\sqrt{p-k-1}+\sqrt{p-1}]}\right] \frac{1}{\sqrt{p}} \\
& \leq K \int_{k+1}^{\infty} \frac{k}{\sqrt{x-k-1} \sqrt{x-1}[\sqrt{x-k-1}+\sqrt{x-1}]} \frac{d x}{\sqrt{x}} \\
& =K \int_{0}^{\infty} \frac{d y}{\sqrt{y} \sqrt{y+k}[\sqrt{y}+\sqrt{y+k}]} \frac{d z}{\sqrt{y+k+1}} \\
& =K \int_{0}^{\infty} \frac{d y}{\sqrt{z} \sqrt{z+1}[\sqrt{z}+\sqrt{z+1}] \sqrt{z+1}} .
\end{aligned}
$$

What is left to estimate is $\left|a_{k}-a_{0}\right| k^{-\frac{1}{2}}+\left|a_{k+1}-a_{1}\right|(k+1)^{-\frac{1}{2}}$, which is obviously bounded, because so are $a_{k}$ and $a_{k+1}$. We have shown (22), therefore (23). According to (21),

$$
\begin{aligned}
m\left(\left\{y \notin \bigcup_{j} 2 B_{j} \backslash \sum_{i}\left|T\left(I-P^{k_{i}}\right) b_{i}(y)\right|>\frac{\lambda}{2}\right\}\right) & \leq \frac{1}{\lambda} C \sum_{i}\left\|b_{i}\right\|_{1} \\
& \leq \frac{C}{\lambda}\|f\|_{1} .
\end{aligned}
$$

Theorem 1 is completely proved. 


\section{A counterexample for $p>2$}

We intend to explain that Theorem 1 is false for $p>2$. For $n \geq 2$, let $\Gamma_{n}$ be the graph formed by two copies of $Z^{n}$ linked between with an edge. Define, for any $x \in \Gamma_{n}, m(x)$ as being the number of neighbours of $x$. Actually, $m(x)$ is equal to $2 n$, except for the two points linked by an edge for which $m(x)=2 n+1$. Equip $\Gamma$ with the measure $m$. On this graph, there exists a constant $C>0$ such that, for any $x \in \Gamma_{n}$ and any $r>0$,

$$
C^{-1} r^{n} \leq V(x, r) \leq C r^{n} .
$$

For any $p>n$ and any function $f$ finitely supported in $\Gamma_{n}$, one has, for any $x, y \in \Gamma$,

$$
|f(x)-f(y)| \leq C d(x, y)^{1-\frac{n}{p}}\|\nabla f\|_{p}
$$

Moreover, for any function $f$ finitely supported in $\Gamma_{n}$, the following Nash inequality holds:

$$
\|f\|_{2}^{1+\frac{2}{n}} \leq C\|f\|_{1}^{\frac{2}{n}}\|\nabla f\|_{2} .
$$

For $n \geq 3$, this Nash inequality is equivalent to the Sobolev inequality (see [3]):

$$
\|f\|_{\frac{2 n}{n-2}} \leq C\|\nabla f\|_{2} .
$$

Define $p$ as being the kernel of the standard random walk on $\Gamma_{n}$ (see [7], p.148). Then, $p$ is a Markov kernel, reversible with respect to $m$, and satisfies (5) and (6). The Nash inequality (26) shows that, for any $x \in \Gamma$ and any $k \in \mathrm{N}^{*}$,

$$
p_{k}(x, x) \leq C k^{-\frac{n}{2}},
$$

(see [4]). Theorem 1.1 in [8] shows that, for any $x, y \in \Gamma_{n}$ and any $k \in \mathrm{N}^{*}$,

$$
p_{k}(x, y) \leq C k^{-\frac{n}{2}} \exp \left[-\frac{d^{2}(x, y)}{C k}\right] .
$$

One also has, for any $x \in \Gamma$ and any $k \in \mathrm{N}^{*}$,

$$
p_{k}(x, x) \geq C k^{-\frac{n}{2}} .
$$

This result follows from Theorem 4.6 of [7].

Assume that $p>n$ and that the Riesz transform on $\Gamma$ is $L^{p}$-bounded. Then, for any $f$ finitely supported,

$$
\|\nabla f\|_{p} \leq C_{p}\left\|(I-P)^{\frac{1}{2}} f\right\|_{p} .
$$


Fix $z \in \Gamma$ and apply (25) and (31) to $f(x)=p^{k}(x, z)$. One gets

$$
\left|p_{k}(x, z)-p_{k}(y, z)\right| \leq C_{p} d(x, y)^{1-\frac{n}{p}}\left\|(I-P)^{\frac{1}{2}} p^{k}(., z)\right\|_{p} .
$$

Choose an integer $l \sim \frac{k}{2}$ and write that

$$
(I-P)^{\frac{1}{2}} p_{k}(., z)=(I-P)^{\frac{1}{2}} P^{l} p_{l}(., z),
$$

which implies that

$$
\left\|(I-P)^{\frac{1}{2}} p_{k}(., z)\right\|_{p} \leq\left\|(I-P)^{\frac{1}{2}} P^{l}\right\|_{p \rightarrow p}\left\|p_{l}(., z)\right\|_{p} .
$$

But the analyticity of $P$ implies that

$$
\left\|(I-P)^{\frac{1}{2}} P^{l}\right\|_{p \rightarrow p} \leq \frac{C}{l^{\frac{1}{2}}} .
$$

(Recall that $P$ is analytic on $L^{p}$ for any $\left.p \in\right] 1,+\infty[$, because $P$ is submarkovian and analytic on $L^{2}$, cf [9], p. 426). Moreover,

$$
\begin{aligned}
\left\|p_{l}(., z)\right\|_{p} & \leq\left\|p_{l}(., z)\right\|_{2}^{\frac{2}{p}}\left\|p_{l}(., z)\right\|_{\infty}^{1-\frac{2}{p}} \\
& \leq p_{l}(z, z)^{\frac{1}{p}}\left\|p_{l}(., z)\right\|_{\infty}^{1-\frac{2}{p}} \\
& \leq C l^{-\frac{n}{2}\left(1-\frac{1}{p}\right)} .
\end{aligned}
$$

In the last line, we used (29). Finally,

$$
\left|p_{k}(x, z)-p_{k}(y, z)\right| \leq C_{p} d(x, y)^{1-\frac{n}{p}} k^{-\frac{1}{2}-\frac{n}{2}\left(1-\frac{1}{p}\right)} .
$$

Thanks to (30), one gets, applying (32),

$$
\left|p_{k}(x, z)-p_{k}(y, z)\right| \leq C_{p}\left[\frac{d(x, y)}{\sqrt{k}}\right]^{1-\frac{n}{p}} p_{k}(z, z) .
$$

Choosing $z=x$ in (33) yields

$$
\left|p_{k}(x, x)-p_{k}(y, x)\right| \leq C_{p}\left[\frac{d(x, y)}{\sqrt{k}}\right]^{1-\frac{n}{p}} p_{k}(x, x) .
$$

As a consequence, for $d(x, y)<a \sqrt{k}$ where $a>0$ is small enough,

$$
\left|p_{k}(x, x)-p_{k}(y, x)\right| \leq \frac{1}{2} p_{k}(x, x),
$$

which implies that

$$
p_{k}(y, x) \geq c k^{-\frac{n}{2}}
$$


whenever $d(x, y)<a \sqrt{k}$. Therefore, by a chaining argument analogous to [13], p. 688, one gets that, whenever $d(x, y)<a k$,

$$
p_{k}(x, y) \geq c k^{-\frac{n}{2}} \exp \left[-\frac{d^{2}(x, y)}{C k}\right] .
$$

This estimate, joined to (29), implies that the Harnack inequality, and therefore the Poincare inequality holds on $\Gamma$ (see [11], Theorem 1.7), which is false. Thus, the Riesz transform on $\Gamma$ is not bounded on $L^{p}$ for $p>n$.

\section{Appendix: proof of a density result on semigroups}

Let us show Lemma 1. The proof is made up of three steps. We start with a general result about contracting semigroups on $L^{2}$.

Lemma 8. Let $\left(T_{t}\right)_{t \geq 0}$ be a continuous semigroup of self-adjoint contracting operators on $L^{2}(X)$, A its infinitesimal generator. Then

$$
\lim _{t \rightarrow+\infty} T_{t} f=I(f), \quad \forall f \in L^{2}(X),
$$

where $I(f)$ is the orthogonal projection of $f$ on $\operatorname{ker}(A)$.

Here is the proof of this lemma. Write

$$
L^{2}(X)=\operatorname{ker}(A) \stackrel{\perp}{\oplus} \operatorname{ker}(A)^{\perp} .
$$

This is true because $A$ is closed, so $\operatorname{ker}(A)$ is closed. Let $f$ belong to $L^{2}(X)$. We decompose

$$
f=\underbrace{I(f)}_{\in \operatorname{ker}(A)}+\underbrace{g}_{\perp \operatorname{ker}(A)}
$$

and we notice that, for every $t \geq 0$,

$$
\begin{aligned}
T_{t} I(f)-I(f) & =\int_{0}^{t} A T_{s} I(f) d s \\
& =\int_{0}^{t} T_{s} A I(f) d s \\
& =0 .
\end{aligned}
$$

Therefore, what is left to be shown is the fact that

$$
\lim _{t \rightarrow+\infty} T_{t} g=0 .
$$

We may regard $A$ as an operator from $\operatorname{ker}(A)^{\perp}$ to $\operatorname{ker}(A)^{\perp}$. Considered in this way, $A$ is one-to-one. Since $A$ is normal, there exists a resolution of the identity $E$ on $\sigma(A)$ such that 


$$
A=\int_{\sigma(A)} \lambda d E(\lambda) .
$$

Since $T_{t}$ is contracting, $\left.\left.\sigma(A) \subset\right]-\infty, 0\right]$. Moreover, $A$ is one-to-one, so that $E(\{0\})=0$. Therefore, the dominated convergence theorem shows that

$$
\lim _{t \rightarrow+\infty} \int_{\sigma(A)} e^{2 \lambda t} d E_{g, g}(\lambda)=0 .
$$

The lemma is proved.

We now state the corresponding result for $L^{p}(X)$ :

Lemma 9. Let $\left(T_{t}\right)$ be as in Lemma 8. Assume also that $T_{t}$ contracts $L^{1}(X)$ and $L^{\infty}(X)$. Then one has

$\lim _{t \rightarrow+\infty} T_{t} f=I(f)$ in the $L^{p}$ norm,$\left.\forall p \in\right] 1,+\infty\left[, \quad \forall f \in L^{1}(X) \cap L^{\infty}(X)\right.$.

First, we show that $I(f) \in L^{1}(X) \cap L^{\infty}(X)$. Let $\left(t_{n}\right)$ be a sequence of positive real numbers which converges to 0 . One has $T_{t_{n}} f \rightarrow I(f)$ in $L^{2}(X)$, so that there exists a subsequence of $\left(t_{n}\right)$, which we still call $\left(t_{n}\right)$, such that $T_{t_{n}} f \rightarrow f$ almost everywhere. Therefore, $I(f) \in L^{\infty}(X)$ and Fatou's lemma shows that $I(f) \in L^{1}(X)$. Hence, we just have to write that $T_{t} f-I(f)=T_{t}(f-I(f))$, use Holder's inequality, Lemma 8 and the fact that $\left\|T_{t}(f-I(f))\right\|_{1} \leq\|f-I(f)\|_{1}$ when $1<p<2$ (resp. $\left\|T_{t}(f-I(f))\right\|_{\infty} \leq$ $\left.\|f-I(f)\|_{\infty}\right)$ to prove Lemma 9.

We are now ready to prove Lemma 1 . Let $p \in] 1,+\infty[$. We consider $\left(T_{t}\right)_{t \geq 0}$ as a continuous semi-group on $L^{p}(X)$, and write that

$$
T_{t} f-f=A\left(f_{t}\right) \text { for } f \in L^{p}(X)
$$

where

$$
f_{t}=\int_{0}^{t} T_{s} f d s .
$$

When $t \rightarrow+\infty$, if $f \in L_{0}^{1}(X) \cap L^{\infty}(X), T_{t} f \rightarrow 0$ in the $L^{p}$ norm. Moreover, $f_{t} \in L^{p}(X) \cap D_{p}(A)$. Lemma 1 is proved.

Acknowledgement. I am grateful to T. Coulhon for many useful remarks about this text. 


\section{REFERENCES}

1. D. Bakry, Transformations de Riesz pour les semi-groupes symétriques, in Séminaire de Probabilités XIX, Lecture Notes in Math. 1123 (1985), 130-175.

2. D. Bakry, The Riesz transforms associated with second order differential operators, Seminar on stochastic processes 88, Birkhaüser, 1989.

3. D. Bakry, T. Coulhon, M. Ledoux, L. Saloff-Coste, Sobolev inequalities in disguise, Indiana Univ. Math. J. 124 (1995), 1033-1074.

4. E. Carlen, S. Kusuoka, D. Stroock, Upper bounds for symmetric Markov transition functions, Ann. Inst. H. Poincaré Probab. Statist., suppl. au no 2 (1987), 245-287.

5. M. Christ, Temporal regularity for random walk on discrete nilpotent groups, Journal of Fourier Analysis and Applications, Kahane Special Issue, 141-151, 1995.

6. T. Coulhon, X. T. Duong, Riesz transforms for $1 \leq p \leq 2$, to appear in Trans. Amer. Math. Soc.

7. T. Coulhon, A. Grigor'yan, On-diagonal lower bounds for heat kernels and Markov chains, Duke Math. J. 89 (1977).

8. T. Coulhon, A. Grigor'yan, Random walks on graphs with regular volume growth, to appear in G.A.F.A..

9. T. Coulhon, L. Saloff-Coste, Puissances d'un opérateur régularisant, Ann. Inst. Henri Poincaré Probab. Statist. 26 (1990), 419-436.

10. R. Coifman, G. Weiss, Analyse harmonique non-commutative sur certains espaces homogènes, Lecture Notes in Math. 242 (1971).

11. T. Delmotte, Parabolic Harnack inequality and estimates of Markov chains on graphs, preprint.

12. A. Grigor'yan, Upper bounds of derivatives of the heat kernel on an arbitrary complete manifold, J. Funct. Anal. 127 (1995), 363-389.

13. W. Hebisch, L. Saloff-Coste, Gaussian estimates for Markov chains and random walks on groups, Ann. Probab. 21 (1993), 673-709.

14. L. Saloff-Coste, Analyse sur les groupes de Lie à croissance polynomiale, Ark. Mat. 28 (1990), 315-331.

DÉPARTEMENT DE MATHÉMATIQUES

UNIVERSITÉ DE CERGY-PONTOISE

2, AVENUE ADOLPHE-CHAUVIN, PONTOISE

F-95302 CERGY-PONTOISE CEDEX

FRANCE 